\title{
Minimización de costos de producción y de contaminantes químicos en una planta de fundición de estaño
}

Janis Martínez MiravaL* JuAn CABRejos Salinas** Miguel Mejía Puente***

\section{RESUMEN}

La investigación consistió en el diseño de un modelo matemático que permitiera reducir los costos de producción en una planta de fundición de estaño, lo cual permitiría la reducción de contaminantes. Se tomó como referencia el modelo de Kim y Lewis (1987), que fue adaptado a la empresa bajo estudio. La problemática que motivó esta investigación provino de las insuficientes técnicas cuantitativas para realizar la programación de producción en fundiciones, al no tomar en cuenta el efecto del trabajo de un lote sobre otros lotes. Luego de probar el modelo, los resultados indicaron un ahorro estimado de S/.3 314964 anuales, de $23 \%$ en el uso del horno y un menor contenido de contaminantes químicos.

Palabras clave: Planta de fundición, Programación de la producción, Eficiencia.

Minimisation OF PRODUCtion COSTS AND CHEMICAL POLLUTANTS IN A TIN FOUNDRY PLANT

\section{ABSTRACT}

The investigation consisted in the design of a mathematical model that allows the reduction of production costs in a tin foundry plant, which would lead to the reduction of pollutants. The model proposed by Kim and Lewis (1987), who was adapted to the company under study, was taken as reference. The situation that motivated this investigation was the insufficient quantitative techniques in the production programming around foundry operations, where the effect a batch has over others is not taken into account. After testing the model, the results indicated estimated savings of S/.3 314964 per year, an estimated saving of $23 \%$ in the use of the melting furnace and a decrease in the content of pollutants.

Keywords:Tin foundry plant, Production programming, Efficiency.

\section{INTRODUCCIÓN}

Actualmente, los niveles de exigencia para la fabricación de productos de calidad son cada vez mayores. Es así que numerosos modelos matemáticos han sido desarrollados para ayudar a la toma de decisiones a nivel operativo, lo cual permite gestionar eficientemente los recursos disponibles de una organización para alcanzar los objetivos en términos de cantidad y calidad, considerando las restricciones de operación.

En el caso específico de la fundición de metales, se ha venido empleando, generalmente, tres técnicas para calcular el costo de la configuración de la carga de fundición: el mejor acierto, el costeo y la carga del menor costo. Mientras que la primera de ellas se basa exclusivamente en la experiencia de los fundidores, la segunda representa un cálculo escueto del costo del material empleado para producir una aleación utilizando una calculadora de mano programable o una computadora personal. La tercera técnica utiliza la programación lineal para optar por los recursos apropiados para minimizar el costo de producción de un solo lote.

Las tres técnicas presentan desventajas importantes: a) no toman en cuenta el efecto de la carga para un lote en el trabajo en otro lote; b) no permiten la toma de decisiones para el largo plazo; y c) no consideran que, en diferentes momentos del horizonte de planificación, los productos tienen distintas composiciones químicas.

Por lo tanto, es necesario proponer un modelo matemático para dar soporte a la gerencia de operaciones en la gestión eficiente del uso de los ingredientes durante un horizonte de planificación determinado, de manera que se obtengan los costos de carga mínimos tomando en cuenta las configuraciones de carga de todos los lotes y, así, poder evaluar el costo-beneficio entre los recursos empleados.

En la presente investigación, en primer lugar, se presenta el modelo genérico de Kim y Lewis [5], a partir del cual se ha diseñado el modelo para el caso de estudio. Seguidamente, se muestran las operaciones de fundición y refinación para la producción de estaño puro, luego de lo cual se obtiene un modelo

\footnotetext{
Magíster en Ingeniería Industrial con mención en Gestión de Operaciones - PUCP. E-mail: jamartinezm@pucp.pe

* Magíster en Ingeniería Industrial con mención en Gestión de Operaciones - PUCP. E-mail: juan.cabrejos@pucp.edu.pe

*** Doctor en Ingeniería Industrial - UNMSM

E-mail: miguel.mejia@pucp.edu.pe
} 
definitivo. Finalmente, se explican los resultados obtenidos luego de las pruebas al modelo.

\section{MARCO TEÓRICO}

Según Kim y Lewis [5], con el deseo de lograr una productividad siempre creciente, la industria de fundición debe estar preparada para utilizar técnicas de administración científica. En este sentido, la investigación describe la aplicación de la programación lineal para determinar la mezcla óptima de recursos a seleccionar en una operación de fundición durante un horizonte de planificación determinado. Dado esto, son tres sus propósitos:

1. Desarrollar un modelo de la operación de fundición de forma completa a través de la programación lineal, considerando que una cantidad variada de aleaciones deben producirse durante un horizonte de planificación prolongado.

2. Resolver el problema de programación lineal mediante software comercial.

3. Comparar la eficiencia del modelo propuesto (al cual llaman OSU) con la obtenida por software diseñado para el mismo propósito: hallar el costo mínimo de carga.

En relación con el tercer propósito, con el modelo OSU se pudo lograr ahorros de, aproximadamente, 10 a $15 \%$ en costos en comparación con software convencional destinado al cálculo del costo mínimo de uso de ingredientes para la fundición, el cual calcula la información de un lote a la vez. Los ahorros se deben a que el modelo tiene la habilidad de asignar la chatarra generada internamente en los momentos más oportunos. Dando soporte a este concepto, citamos a Rong y Lahdelma [11]: "Sin duda alguna, el uso de la chatarra ofrece la oportunidad de producir productos de alta calidad de la manera más económica" (traducción propia). Además, Kim y Lewis [5] añaden que es probable que la utilidad del modelo OSU para la toma de decisiones de planificación de largo plazo sea más importante que los ahorros que podrían generarse.

La presente investigación se enfocó en alcanzar el primer propósito mencionado anteriormente con base en la operación de fundición de la empresa FURESA. El modelo fue resuelto utilizando el software Lingo 9.0, pero aplicando la sintaxis de Lindo.

Tradicionalmente se han identificado tres técnicas para calcular el costo de la configuración de la carga de fundición: la "regla del dedo" o del mejor acierto, algún tipo de técnica de costeo $y$, finalmente, el método de la carga del menor costo. Mientras que la primera de ellas -posiblemente, la más común- se basa únicamente en la experiencia del fundidor, la segunda es un cálculo simple del costo del material empleado para producir una aleación utilizando una calculadora de mano programable o una computadora personal. En cambio, la tercera técnica -posiblemente la menos usada- es llamada "algoritmo de menor costo de carga", y utiliza la programación lineal para seleccionar los recursos apropiados para minimizar el costo de producción de un solo lote.

A pesar de que las tres técnicas pueden ser útiles en determinadas ocasiones y hasta cierto alcance, presentan desventajas importantes de las cuales las correspondientes a las dos primeras son las de mayor impacto. Por ejemplo, respecto de la regla del mejor acierto, cuando se confía únicamente en la experiencia del fundidor, con frecuencia, un método que pueda resultar ineficiente podría repetirse más de una vez. Además, en cuanto a las dos primeras técnicas, el mejor acierto y el costeo, ambas sufren del problema de no poder evaluar el nivel de beneficios entre varias alternativas competitivas.

Cuando se trata de la tercera técnica, Kim y Lewis [5] mencionan que esta sufre del hecho de que se debe calcular la carga según los requerimientos de composición química para un lote cada vez, lo cual significa que, generalmente, no incluye el impacto de los retornos futuros, políticas de control de inventarios y exactitud en el cálculo de las cantidades de carga. Sin embargo, agregan lo siguiente:

Este artículo es un estudio de una herramienta de investigación de operaciones aplicada a la operación completa de fundición. Ecuaciones matemáticas fueron formuladas para obtener la asignación de recursos más económicos. Un número de ecuaciones que representan las operaciones de fundición fueron formuladas como restricciones. El objetivo fue minimizar el costo de materiales para la carga no solo para una operación de fundición en el horno, sino para un número de operaciones de fundición para varias aleaciones diferentes sobre un periodo de tiempo u horizonte de planificación específicos. Para lograr esta meta de minimización del costo de la operación de fundición sobre un periodo de tiempo prolongado, el reciclaje de la chatarra generada internamente tiene que estar incluido en el modelo (Traducción propia).

Se debe mencionar que existen tres restricciones básicas que se deben satisfacer, tal y como lo mencionan Kim y Lewis [5]. 
a. Demanda y/o capacidad del horno: no se debe exceder una capacidad determinada de horno ni la demanda del cliente.

b. Disponibilidad de materiales: no se debe exceder el uso de los recursos disponibles en ningún momento en el tiempo.

c. Balance químico del metal: se debe mantener la composición química apropiada en el trabajo de un lote.

Kim y Lewis [5] hacen una revisión bibliográfica de trabajos que precedieron a la elaboración de su artículo. Así, mencionan a Pehlke [9], Mikelonis [6], Giszczak [3], Barrick [2] y la Sociedad Americana de Fundidores (en inglés, American Foundrymen's Society, o AFS) [1]. En relación con los autores citados, Kim y Lewis [5] afirman que sus técnicas proveen una excelente herramienta para que los fundidores puedan determinar configuraciones de carga de manera más eficiente en cuanto al costo. Además, indican que todas estas técnicas están basadas en el enfoque de la programación lineal, pero que, sin embargo, todas sufren del hecho de que son modelos estáticos en cuanto a que consideran el cálculo de la carga de materiales para el trabajo de un lote por vez. En este sentido, presentan las consecuencias al respecto:

- Las técnicas no permiten la toma de decisiones para el largo plazo.

- Las técnicas no toman en cuenta el efecto de la carga para un lote sobre el trabajo en otro lote, por lo que se puede perder eficiencia.

- Las técnicas no consideran que, en diferentes momentos del horizonte de planificación, los retornos tienen distintas composiciones químicas, lo cual puede afectar la eficiencia y la eficacia.

En relación con el último punto, Rong y Lahdelma [11] mencionan en su trabajo que, cuando una carga es fundida, la incertidumbre en la composición química de la chatarra causa un riesgo considerable de que el producto resultante falle en satisfacer los requerimientos de la composición. En este sentido, agregan que hay dos retos en la optimización de la carga de chatarra en el ámbito del acero:

- Los constituyentes de la chatarra y los productos de acero son diversos. Dependiendo del proceso de fundición y de los requerimientos del producto particular, algunos constituyentes de la chatarra son considerados impurezas, mientras que otros, aditivos valiosos. Por lo tanto, es esencial que una mezcla de tipos apropiados de chatarra en proporciones correctas sea seleccionada para cada producto.

- Los diversos materiales de chatarra son, por lo general, separados en clases diferentes; sin embargo, los materiales dentro de una misma clase pueden ser, incluso, muy heterogéneos. Esto significa que existen grandes desviaciones en las propiedades de los materiales en cada clase. Por lo tanto, se hace complicado realizar análisis de composiciones químicas exactas para cada tipo de chatarra, y esta incertidumbre se propaga a la mezcla de chatarras que van a la carga de fundición.

Volviendo al trabajo de Kim y Lewis [5], los autores indican que Pehlke [9] fue el primero en presentar la optimización de carga de cúpula a través de la formulación matemática de las tres restricciones básicas mencionadas anteriormente y el planteamiento de una ecuación de costo. Luego, la programación lineal fue aplicada para resolver el problema. "La formulación de Pehlke bien representa la naturaleza general del problema del menor costo de carga" (Kim y Lewis 1987, p. 736, traducción propia). Una ventaja citada de este programa es que puede ejecutarse fácilmente en una computadora personal; sin embargo, su desventaja es no poder manejar problemas de largo alcance ni incluir ratios de recuperación de chatarra y uso de material en la formulación.

Sobre Mikelonis [6], los autores indican que aplicó la programación lineal en un intento de obtener una carga del menor costo para la operación de fundición de cúpula. Su formulación matemática era similar a la de Pehlke, sin embargo, utilizó un sistema que le permitió trabajar con un problema de mayor alcance. Aun así, al igual que en el trabajo de Pehlke, su enfoque quedaba restringido a optimizar operaciones simples de fundición.

En cuanto a Giszczak [3], los autores señalan que su formulación, que reúne la demanda de ciertos tipos de aleaciones y las trata como una sola, se basó en una idea similar a las formulaciones de Pehlke [9] y Mikelonis [6], pero con un enfoque más global. Entre las ventajas citadas se encuentran la mejor comunicación entre departamentos, la posibilidad de centralizar las decisiones de compra mediante la selección del vendedor más económico y, por último, la posibilidad de planificar la producción hasta cierta extensión. Sin embargo, si algunos materiales se volvían no disponibles de repente, el resultado de la programación lineal debía examinarse o recalcularse, dada la estructura de la formulación matemática. 
Por otro lado, los autores afirman que Barrick (1971) aplicó la programación lineal como parte de un intento por establecer una tienda de fundición computarizada, y creó un modelo que también resolvió el problema del menor costo de carga para un lote a la vez. No obstante, esto no sería suficiente para la planificación de la utilización de los recursos en el largo plazo. Entre las ventajas citadas se encuentran la integración entre el departamento de fundición y de control de inventarios a través de una interfaz en tiempo real entre ambas áreas, la facilidad de uso debida al aspecto interactivo del programa, la posibilidad de conexión con la producción y la consideración del ratio de recuperación.

Finalmente, Kim y Lewis nos hablan del trabajo de la AFS (1986), que había lanzado un paquete de software que calculaba la carga de menor costo. Dicho software incluía la pérdida de material durante la fundición en la formulación de la carga de menor costo. Según los autores, la formulación de la AFS es, básicamente, la misma que introdujo Pehlke (1973). La ventaja principal es que el paquete de software parece haber sido diseñado para una computadora personal y, por lo tanto, aplicable para compañías de fundición pequeñas. Entre otras ventajas citadas se encuentran las siguientes: el operario de fundición podía transportar una computadora personal donde le fuera conveniente y ejecutar el algoritmo de carga de menor costo cuando fuese necesario, la formulación consideraba la pérdida de material durante la fundición, se podía efectuar un mantenimiento conveniente del sistema y se proveía un medio simple para el control de inventarios.

De la misma manera, se citan algunas desventajas: la planificación de la producción en el largo plazo se hacía ineficiente dado que el programa solo calculaba la carga de menor costo para un lote a la vez; el cálculo de cantidades de carga con un buen nivel de exactitud se hacía complicado, ya que la capacidad computacional del programa era limitada; y debido a que solo se podían calcular las cantidades de carga para un lote a la vez, el control de inventarios, las compras y las decisiones de planificación de largo plazo eran limitadas.

En conclusión, Kim y Lewis (1987, 736-737) afirman que: "Con el fin de tener un enfoque más global o dinámico hacia la minimización general del costo de carga, todas las configuraciones de carga de todos los lotes durante un horizonte de planificación deberían ser calculadas simultáneamente para evaluar el costo-beneficio entre los recursos materiales en competencia. Este es, entonces, el objetivo de esta investigación" (traducción propia).

\section{ESTUDIO DE CASO}

La empresa FURESA se encarga, principalmente, de producir estaño metálico, el cual es empleado como materia prima para otros procesos industriales fuera del país. En este sentido, la empresa es capaz de fabricar productos de estaño de alta pureza, los cuales pueden llegar a tener un contenido mínimo de $99.94 \%$ en estaño y máximo de $0.01 \%$ en plomo (Minsur 2011a).

Los procesos principales que realiza FURESA son la fundición y la refinación. En relación con la operación de fundición, la mayoría de los ingredientes utilizados son transportados en camiones a la planta y pasan por un control inicial de calidad, mientras que otros, en menor proporción, se generan a nivel interno como subproductos para recirculación (retornos). Luego, los ingredientes son trasladados por medio de fajas transportadoras a ocho tolvas, desde donde quedan listos para su empleo.

De los ingredientes utilizados, el más importante es el concentrado de estaño, que existe en dos clases: gravimétrico y de flotación, y se trata de óxido de estaño con un contenido aproximado de estaño metálico en peso de 60\% (considerando ambas clases), mientras que el $40 \%$ restante se reparte entre otras especies minerales acompañantes. El concentrado es recibido diariamente en bolsas de 1.5 toneladas. La empresa cuenta con un almacén que en la actualidad administra 11000 toneladas de concentrado y 12000 toneladas de carbón. Además, existe una zona de descarga específica donde se registra el peso de los ingredientes ingresantes y se controla su calidad.

El carbón empleado en la planta es $100 \%$ peruano y se agrega al horno en dos momentos distintos: primero, durante la etapa de fusión y, luego, durante la etapa de reducción, para la producción de un lote de metal crudo líquido. Por otro lado, desde un centro de control se definen las cantidades a utilizar de cada ingrediente para el trabajo de cada lote, además de controlarse los diversos parámetros termodinámicos (temperaturas, presiones, etc.).

En la planta se cuenta con dos hornos para evitar los tiempos muertos. Es decir, después de utilizar un horno para producir una determinada cantidad de lotes de metal crudo, aquel debe recibir un mantenimiento apropiado (básicamente, reemplazo de los ladrillos refractarios) antes de volver a ser usado. Así, cuando llega el momento de realizar el mantenimiento del primer horno, el segundo horno está disponible para su empleo. 
El proceso de fundición se divide en campañas que se identifican por letras ( $A, B, C$, etc.). Estas, a su vez, se dividen en lotes que se identifican por la letra de la campaña y el número ordinal específico (A001, A002, etc.). La duración de una campaña va desde el inicio del funcionamiento de un horno hasta que es detenido para su mantenimiento. Por otro lado, un solo lote abarca las etapas de fusión, reducción, granulación y limpieza. En promedio, en un día se trabajan cuatro lotes.

\section{a. La operación de fundición}

En la operación de fundición se realiza, fundamentalmente, la conversión de los concentrados de estaño junto con otros ingredientes (fundentes, elementos recirculantes y otros) en un metal fundido líquido que posee, al finalizar la operación, una concentración promedio de 98\% de estaño. Esta conversión se realiza a través de un proceso químico-metalúrgico denominado "reducción" y que tiene lugar en el horno. El proceso de fundición abarca el trabajo de los lotes y campañas; sin embargo, podemos visualizarlo prestando atención a las cuatro etapas de un lote: fusión, reducción, granulación y limpieza. Mientras que en las etapas de fusión y reducción se realizan las reacciones químicas principales para formar el metal crudo líquido, la granulación se refiere al proceso mediante el cual la escoria expulsada del horno es confrontada con un chorro de agua fría $y$, luego, dejada en reposo en una piscina para la su formación en granos. Finalmente, la limpieza es un mantenimiento parcial del horno que está siendo utilizado, en la cual se verifica que todos sus componentes se encuentren en buen estado, así como los componentes del bag-house, o colector de polvos, que se encuentra en la parte superior, entre otras tareas. En general, el trabajo de un lote puede llegar a durar hasta siete horas.

Los ingredientes para la fundición son los siguientes: concentrado gravimétrico de estaño, pellets de la mezcla de concentrado de flotación de estaño y polvo de estaño, carbón (usado en la etapa de fusión y de reducción), mineral de fierro, dross de fierro, caliza y escoria marginal. Estos ingredientes se agregan al horno de fundición en cantidades específicas para poder alcanzar los requerimientos de calidad del metal crudo que pasará a la refinación. La empresa FURESA suele trabajar con una capacidad cercana a la máxima, considerando la alta demanda constante de productos de estaño puro (lingotes, granallas, hojuelas) y otras aleaciones de estaño que recibe. Los hornos que utiliza llevan el nombre de Ausmelt, que le permiten lograr una alta eficiencia en la operación de fundición debido a su tecnología interna.

\section{b. La operación de refinación}

La planta de la empresa FURESA tiene una capacidad de procesamiento promedio de 65,000 toneladas de concentrado por año, a partir de lo cual se obtiene un aproximado de 40,000 toneladas de estaño refinado (Minsur 2011b; Pinedo 2009).

En cuanto a la refinación de metales, Heshmatpour y Copeland [4] realizaron investigaciones sobre el uso de la refinación por fundición para descontaminar desechos metálicos, para lo cual el conocimiento de los aspectos metalúrgicos del proceso es esencial. Una de sus conclusiones fue que el plomo, el estaño, el zinc y las aleaciones estaño-plomo mostraron un excelente potencial para descontaminación, particularmente, mediante el uso de escorias de bajo punto de fusión y la fundición en un horno de resistencia eléctrica. En sí, según los autores citados, todos los metales que llegaron a estudiar podrían ser descontaminados efectivamente a través de la selección apropiada de la técnica de fundición y las escorias.

El término "descontaminación" puede utilizarse para describir lo que se realiza en la operación de refinación de la empresa FURESA, cuya planta cuenta con siete ollas de refinación, con una capacidad de 50 toneladas cada una, por medio de las cuales se efectúa la operación de refinación en secuencia. Esta operación comprende cuatro pasos: 1) remoción de fierro, 2) remoción de cobre, 3) remoción de arsénico y antimonio, y 4) remoción de aluminio. Cuando es necesario, luego de estos cuatro pasos, para la remoción de plomo y bismuto, se utilizan cristalizadores electro-térmicos.

Finalmente, la empresa FURESA, aparte de los productos principales que produce, que son los lingotes, las granallas y las hojuelas, como resultado de su trabajo en la planta de subproductos, produce aleaciones estaño-plomo, peltre y aleaciones estaño-cobre.

\section{MODELO MATEMÁTICO}

\section{a. Suposiciones básicas}

Para el desarrollo del modelo matemático se hicieron los supuestos siguientes:

1. Un periodo 30 días es suficiente para probar la funcionalidad del modelo matemático.

2. Los dos hornos de la planta de fundición funcionan como uno solo que trabaja continuamente (sin detenciones por mantenimiento entre campañas). 
3. Para el horizonte de planificación, se conocen, o pueden aproximarse, las sumas totales de ingredientes a agregar al horno para la producción de cada lote de metal crudo líquido y las disponibilidades de cada ingrediente.

4. Se puede aproximar el ratio de recuperación de cada elemento químico.

5. Los polvos de estaño, a pesar de que no son agregados como tales directamente al horno sino mediante su mezcla con el concentrado de flotación bajo la forma de pellets, son un ingrediente recirculante para el modelo matemático.

6. Al inicio de la prueba del modelo matemático no se dispone de polvos como chatarra interna o recirculantes (retornos).

7. El dross de fierro, a pesar de que en cierta medida contiene cantidades de elementos que recirculan a la planta de fundición, es un ingrediente no recirculante, que no es considerado chatarra interna para el modelo matemático.

\section{b. Formulación del modelo matemático}

El modelo matemático diseñado a partir de la adaptación del modelo de Kim y Lewis (1987) a la operación de fundición -utilización de ingredientes para la producción de metal crudo líquido de estaño- de la empresa FURESA, se describe a continuación:

\section{Parámetros}

$C P_{i}$ : Costo en nuevos soles por tonelada para el uso del ingrediente i.

CS: Costo en nuevos soles por tonelada para el uso del ingrediente I.

$\mathrm{CH}_{\mathrm{m}}$ : Costo en nuevos soles por tonelada para el uso del ingrediente $\mathrm{m}$.

$C C_{n}$ : Costo en nuevos soles por tonelada para el uso del combustible $n$.

$\operatorname{Max}_{(j, k)}$ : Límite máximo de toneladas de ingredientes que se considera para el lote j en el día k en cuanto a la capacidad del horno.

$D X_{(\mathrm{i}, \mathrm{k})}$ : Cantidad de toneladas disponibles del ingrediente i destinadas para el día k.

$D Y_{(i, k)}$ : Cantidad de toneladas disponibles del ingrediente I destinadas para el día k.

$D Z_{(\mathrm{m}, \mathrm{k})}$ : Cantidad máxima de toneladas disponibles del ingrediente $\mathrm{m}$ para la producción del día k.
$R_{\mathrm{m}}$ : Tiempo de retorno para el ingrediente $\mathrm{m}$ en días.

$R e c_{m}$ : Porcentaje de cada lote que es procesado para formar el ingrediente $\mathrm{m}$.

$D C_{n, k}$ : Cantidad de toneladas disponibles del combustible $\mathrm{n}$ en el día $\mathrm{k}$.

$W_{\mathrm{h}}$ : Ratio de recuperación en porcentaje del elemento químico $\mathrm{h}$.

$h$ : Índice de elementos o compuestos químicos presentes en la producción del lote $\mathrm{j}, \mathrm{h}=1,2, \ldots$, $Q, Q$ indica el número máximo de elementos químicos presentes.

$A P_{\mathrm{h}, \mathrm{i}}$ : Cantidad en porcentaje del elemento químico $\mathrm{h}$ contenido en el ingrediente $\mathrm{i}$.

$A D_{\mathrm{h}, \mathrm{l}}$ : Cantidad en porcentaje del elemento químico $h$ contenido en el ingrediente $\mathrm{I}$.

$A S_{h, m}$ : Cantidad en porcentaje del componente químico $\mathrm{h}$ contenido en el ingrediente $\mathrm{m}$.

$L S_{\mathrm{h}, \mathrm{j}, \mathrm{k}}$ : Límite superior en porcentaje del elemento químico h para producir el lote j el día $\mathrm{k}$.

$E_{\mathrm{j}, \mathrm{k}}$ : Cantidad mínima de toneladas de estaño a producir en lote j del día k.

$r_{i}$ : Ratio con que el ingrediente i es usado en relación con la cantidad total de toneladas de concentrado gravimétrico, concentrado de flotación y polvo de estaño agregadas a un lote. $\mathrm{i}=1,2$.

$s_{i}$ : Ratio con que el ingrediente $\mathrm{i}$ es usado en relación con la cantidad de carga total de un lote. $i=3,4$.

$p_{r}$ : Proporción promedio con que se usa el carbón para reducción en un lote respecto de su carga total.

$p_{f}$ : Proporción promedio con que se usa el carbón para fusión en un lote respecto de su carga total.

$f_{n}$ : Ratio de utilización del combustible $\mathrm{n}$ definido como la cantidad usada de toneladas de dicho combustible por tonelada de carga total de ingredientes agregados al horno.

\section{Variables de decisión}

$X_{i, j, k}$ : Cantidad de toneladas del ingrediente i (tipo 1: favorece la reducción) usadas para el lote j en el día $\mathrm{k}$.

i: Índice de ingredientes del tipo 1. i = 1, 2, .., L; $L$ es el número total de este tipo de ingredientes.

$j$ : Índice del número de lote. $\mathrm{j}=1,2, \ldots, M_{k} ; M_{k}$ es el número total de lotes del día $k$. 
$k$ : Índice del número de día. $k=1,2, \ldots, N ; N$ es el número total de días del horizonte de planificación.

$Y_{1, j, k}:$ Cantidad de toneladas del ingrediente I (tipo 2: agrega estaño a la mezcla) usadas para el lote $j$ en el día $k$.

I: Índice de ingredientes del tipo $2 . \mid=1,2, \ldots, 0 ; 0$ es el número total de estetipo de ingredientes.

$Z_{m, j, k}:$ Cantidad de toneladas del ingrediente $m$ (tipo 3: es recirculante y aporta estaño) usadas para el lote $j$ en el día $k$.

$m$ : Índice de ingredientes del tipo $3 . m=1,2, \ldots, T$; $T$ es el número total de estetipo de ingredientes.

$V_{\mathrm{n}, \mathrm{j}, \mathrm{r}}:$ Cantidad de toneladas del combustible usadas para el lote j en el día $\mathrm{k}$.

$n$ : Índice de combustibles. $n=1,2, \ldots, C ; C$ es el número total de combustibles.

$N_{\mathrm{j}, \mathrm{k}}$ : Cantidad total de toneladas de ingredientes ingresada al horno para el lote j en el día $\mathrm{k}$.

$H_{\mathrm{j}, \mathrm{k}}$ : Cantidad total de toneladas agregadas al horno de concentrado gravimétrico, concentrado de flotación y polvo de estaño en el lote $j$ del día $k$.

\section{Función objetivo}

Minimizar

$$
\begin{array}{r}
\sum_{i=1}^{L} \sum_{j=1}^{M_{k}} \sum_{k=1}^{N} C P_{i} X_{i, j, k}+\sum_{l=1}^{O} \sum_{j=1}^{M_{k}} \sum_{k=1}^{N} C S_{l} Y_{l, j, k}+ \\
\sum_{m=1}^{T} \sum_{j=1}^{M_{k}} \sum_{k=1}^{N} C H_{m} Z_{m, j, k}+\sum_{n=1}^{C} \sum_{j=1}^{M_{k}} \sum_{k=1}^{N} C C_{n} V_{n, j, k}
\end{array}
$$

\section{Restricciones}

Restricciones de capacidad

$$
\begin{aligned}
& \sum_{i=1}^{L} X_{i, j, k}+\sum_{l=1}^{O} Y_{l, j, k}+\sum_{m=1}^{T} Z_{m, j, k}-N_{j, k}=0, \text { para todo } j, k \\
& N_{j, k} \leq \operatorname{Max}_{j, k}, \text { para todo } j, k
\end{aligned}
$$

Restricciones de disponibilidad para los ingredientes del tipo 1

$$
\sum_{j=1}^{M_{k}} X_{i, j, k} \leq D X_{i, k}, \text { para todo } i, k
$$

Restricciones de disponibilidad para los ingredientes del tipo 2

$$
\sum_{j=1}^{M_{k}} Y_{l, j, k} \leq D Y_{l, k}, \text { para todo } l, k
$$

Restricciones de disponibilidad para los ingredientes del tipo 3

$$
\begin{aligned}
& \sum_{k^{\circ}=1}^{k} \sum_{j=1}^{M_{k}} Z_{m, j, k} \cdot \leq Z_{m, k}, \text { para todo } m, k \\
& D Z_{m, k}=\sum_{p=1}^{k-R_{m}} \sum_{j=1}^{M_{k}} N_{j, p} R e c_{m} \text {, para todo } k>R_{m} \\
& D Z_{m, k}=0, \text { para todo } k \leq R_{m}
\end{aligned}
$$

Restricciones de disponibilidad para los combustibles

$$
\sum_{j=1}^{M_{k}} V_{n, j, k} \leq D C_{n, k}, \text { para todo } n, k
$$

Restricciones para la formación de pellets

$$
Y_{2, j, k}-Z_{1, j, k}=0 \text {, para todo } j, k
$$

Restricciones de balance químico para el cumplimiento de límites

$\sum_{i=1}^{L} W_{h} A P_{h, i} X_{i, j, k}+\sum_{l=1}^{o} W_{h} A D_{h, l} Y_{l, j, k}+\sum_{m=1}^{T} W_{h} A S_{h, m} Z_{m, j, k}$

$\leq L S_{h, j, k}\left(0.5 N_{j, k}\right)$, para todo $h, j, k$

$\sum_{i=1}^{L} W_{h} A P_{h, i} X_{i, j, k}+\sum_{l=1}^{O} W_{h} A D_{h, l} Y_{l, j, k}+\sum_{m=1}^{T} W_{h} A S_{h, m} Z_{m, j, k}$

$\geq E_{j, k}$, para todo $h, j, k$

Restricciones para el empleo de ingredientes del tipo 1

$\mathrm{Y}_{1, \mathrm{j}, \mathrm{k}}+\mathrm{Y}_{2 \mathrm{j,k}, \mathrm{k}}+\mathrm{Z}_{1, \mathrm{j}, \mathrm{k}}-\mathrm{H}_{\mathrm{j}, \mathrm{k}}=0$, para todo $j, k$

$\mathrm{X}_{\mathrm{i}, \mathrm{j}, \mathrm{k}}-\mathrm{r}_{\mathrm{i}} \mathrm{H}_{\mathrm{j}, \mathrm{k}} \geq 0$, para $i=1,2$ y todo $j, k$ 
$\mathrm{X}_{\mathrm{i}, \mathrm{j}, \mathrm{k}}-\mathrm{s}_{\mathrm{i}} \mathrm{N}_{\mathrm{j}, \mathrm{k}} \geq 0$, para $\mathrm{i}=3,4$ y todo $j, k$

$\mathrm{p}_{\mathrm{r}} \mathrm{X}_{1, \mathrm{j}, \mathrm{k}}-\mathrm{p}_{\mathrm{f}} \mathrm{X}_{2, \mathrm{j}, \mathrm{k}} \geq 0$ ", para todo $j, k$

Restricciones para el empleo de los combustibles

$\mathrm{V}_{\mathrm{n}, \mathrm{j}, \mathrm{k}}-\mathrm{f}_{\mathrm{n}} \mathrm{N}_{\mathrm{j}, \mathrm{k}} \geq 0$, para todo $n, j, k$

\section{ANÁLISIS DE RESULTADOS}

La comparación entre los resultados estimados de la empresa y los resultados obtenidos con el modelo matemático se realizará desde dos puntos de vista: el económico y la presencia de contaminantes químicos en el producto final de la etapa de fundición.

\section{a. Análisis económico}

Los pasos seguidos para calcular el costo total estimado de la operación de fundición para un horizonte de planificación de 30 días se muestran a continuación.

En primer lugar se calculan los costos estimados de la empresa:

1. Actualmente, en promedio, una cantidad total de 113.20 toneladas de ingredientes ingresa al horno para el trabajo de un lote.

2. Aplicando los porcentajes de contenido metálico de estaño proporcionados por la empresa a las cantidades de ingredientes utilizadas actualmente (que suman en total las 113.20 toneladas mencionadas), se tiene que, de las 113.20 toneladas de carga, un total de 45.61 toneladas corresponde al estaño ingresante para un lote.

3. Si se emplea un ratio de recuperación de $98 \%$ para el estaño (basado en el nivel de pureza del metal líquido que se obtiene luego de la fundición), se obtiene que la cantidad estimada final de estaño que se produce es de 44.70 toneladas para un lote.

4. Para hallar el costo total del uso de ingredientes para el trabajo de un lote, multiplicamos los costos de los ingredientes por las cantidades respectivas de ingredientes que conforman la carga de 113.20 toneladas y sumamos los resultados, con lo cual se obtiene un costo de S/. 129368 por lote.

5. Paraincluirlos costos delempleo decombustibles, utilizamos los ratios proporcionados por la empresa, así como el total señalado en el paso 1, para obtener S/. 10401 por lote; luego, se añade la cantidad calculada en el paso 4 para obtener un valor de producción de S/. 139769 por lote.

6. Como la cantidad de lotes a producir en el horizonte de planificación es de 120 (4 lotes por día durante 30 días), multiplicamos el costo total por lote del paso 5 por este número y hallamos que el costo total estimado de la operación es S/. 16772259.

En segundo lugar, se calculan los costos obtenidos según el modelo matemático:

1. Como la cantidad estimada de estaño producido para un lote es 44.70 toneladas, este mismo valor es el que se utiliza en el modelo matemático como dato de entrada para los 120 lotes del horizonte de planificación (ver variable), todo con la finalidad de mantener una misma base. El resultado de la ejecución del modelo es S/.137 467, incluyendo el uso de combustibles.

2. Finalmente, multiplicamos la cantidad calculada en el paso anterior por 120 (número total de lotes para el horizonte de planificación) y hallamos que el costo total estimado de la operación es S/. 16496012.

Ahora se puede concluir lo siguiente:

- Se obtiene un ahorro mensual estimado de S/. 276247 en cuanto al costo total de la operación, lo cual representa una reducción de $1.65 \%$, respecto de la operación actual.

- La meta de 44.70 toneladas de estaño se está logrando con 87.23 toneladas de carga total en el modelo matemático, a diferencia de las 113.20 toneladas de la operación actual, lo cual se debe, principalmente, a la diferencia de toneladas que están siendo agregadas de escoria marginal y de concentrado gravimétrico en ambos casos. Los resultados del modelo arrojan que no se debe usar escoria marginal, mientras que en la operación actual se está empleando una cantidad cercana a la mitad de lo que se agrega de concentrado gravimétrico (se emplea más de 50 toneladas de este concentrado). En otras palabras, se obtiene un ahorro de $23 \%$ en el uso del horno.

\section{b. Análisis de contaminantes}

En relación con la presencia de contaminantes químicos, se realiza una comparación similar, es decir, se contrastan los resultados del proceso actual en la empresa con los del modelo matemático. El análisis considera los componentes químicos de los ingredientes, excepto el estaño. En la Tabla 1 se muestran las cantidades de toneladas estimadas 
de componentes químicos empleados tanto en la operación actual como en el modelo matemático, así como las diferencias existentes.

De la Tabla 1 se puede afirmar que:

- El contenido de contaminantes para el metal crudo según la combinación de ingredientes del modelo matemático es menor que para la operación actual. Principalmente, se observa mayor diferencia en los contenidos de antimonio, fierro y arsénico, lo que implica procesos menos intensos de remoción durante la etapa de refinación del metal crudo en la propuesta del modelo matemático.

- El menor contenido de contaminantes en la propuesta del modelo matemático implica menores costos, menor uso de energía, y menor tiempo de procesamiento para la remoción de contaminantes en la refinación. Estos ahorros, si bien son identificables, no han sido medidos ya que se ubican fuera del ámbito de nuestra investigación.

Tabla 1. Comparación sobre presencia de contaminantes

\begin{tabular}{|c|c|r|r|r|c|}
\hline $\begin{array}{c}\text { Componentes } \\
\text { químicos }\end{array}$ & $\begin{array}{c}\text { Ratio de } \\
\text { recuperación }\end{array}$ & $\begin{array}{c}\text { Presencia: } \\
\text { actual (t) }\end{array}$ & $\begin{array}{c}\text { Presencia: } \\
\text { modelo (t) }\end{array}$ & $\begin{array}{c}\text { Diferencia a favor } \\
\text { (t) }\end{array}$ & Diferencia (\%) \\
\hline $\mathrm{Fe}$ & $4 \%$ & 0.231354 & 0.095661 & 0.135693 & $58.65 \%$ \\
\hline $\mathrm{As}$ & $4 \%$ & 0.008860 & 0.003746 & 0.005114 & $57.72 \%$ \\
\hline $\mathrm{Cu}$ & $4 \%$ & 0.000453 & 0.000399 & 0.000054 & $11.83 \%$ \\
\hline $\mathrm{Sb}$ & $4 \%$ & 0.003165 & 0.001012 & 0.002152 & $68.01 \%$ \\
\hline $\mathrm{Pb}$ & $4 \%$ & 0.000569 & 0.000455 & 0.000113 & $19.95 \%$ \\
\hline $\mathrm{CaO}$ & $0 \%$ & 0.000000 & 0.000000 & 0.000000 & \\
\hline $\mathrm{Al} \mathrm{O}_{3}$ & $0 \%$ & 0.000000 & 0.000000 & 0.000000 & 0.000000 \\
\hline $\mathrm{SiO}$ & $0 \%$ & 0.000000 & 0.000000 & 0.000000 & \\
\hline $\mathrm{S}$ & $0 \%$ & 0.000000 & 0.000000 & 0.000000 & \\
\hline $\mathrm{C}$ & $0 \%$ & 0.000000 & 0.000000 & 0.003893 & $40.62 \%$ \\
\hline $\mathrm{Zn}$ & $4 \%$ & 0.009584 & 0.005691 & 0.000578 & $40.62 \%$ \\
\hline $\mathrm{In}$ & $4 \%$ & 0.001424 & 0.000846 & 0.000000 & \\
\hline $\mathrm{FeO}$ & $0 \%$ & 0.000000 & 0.000000 & 0.000000 & \\
\hline $\mathrm{MgO}$ & $0 \%$ & 0.000000 & 0.000000 & 0.000000 & \\
\hline $\mathrm{P}$ & $0 \%$ & 0.000000 & 0.000000 & & \\
\hline
\end{tabular}

Fuente: Elaboración propia de tabla basada en los resultados del modelo.

\section{CONCLUSIONES}

Las principales conclusiones de la investigación son las siguientes:

- Los resultados económicos conseguidos indican un ahorro mensual de S/. 276247 usando el modelo matemático, con base en el trabajo de 120 lotes (30 días), lo cual equivale a un ahorro anual de S/. 3314964 .

- El modelo matemático alcanza el objetivo de producir 44.70 toneladas de estaño con una menor cantidad de toneladas de carga total, lo cual significa un ahorro de $23 \%$ en el empleo del horno de fundición.

- El contenido de contaminantes para el metal crudo según la combinación de ingredientes del modelo matemático es menor que para la operación actual. Principalmente, se observa mayor diferencia en los contenidos de antimonio, fierro y arsénico, lo que implica un menor trabajo de remoción durante la etapa de refinación del metal crudo. 
- El menor contenido de contaminantes en la propuesta del modelo matemático implica menores costos, menor uso de energía, y menor tiempo de procesamiento para la remoción de contaminantes en la refinación.

\section{REFERENCIAS BIBLIOGRÁFICAS}

[1] American Foundrymen's Society (1986). Least cost charge. AFS Transactions.

[2] Barrick, J. (1971). Computerized melt shops for greater profits. AFS Transactions. Vol. 130.

[3] Giszczak, T. (1973). Least cost raw material acquisition-MINICOST. AFS Transactions. Vol. 129.

[4] Heshmatpour, B. y Copeland, G. (1981). Metallurgical aspects of waste metal decontamination by melt refining. Nuclear and Chemical Waste Management. Vol. 2, N. ${ }^{\circ} 1,25-31$.

[5] Kim, J. y Lewis, R. (1987). A large scale linear programming application to least cost charging for foundry melting operations. Transactions of the American Foundrymen's Society. Vol. 95, 735-744.
[6] Mikelonis, P. (1979). Least cost cupola charging through linear programming. AFS Transactions. Vol. 170.

[7] Minsur (2011a). Nuestros productos - el estaño. www.minsur.com.pe/estano.htm (Visitado el 0504-2011).

[8] Minsur (2011b). Nuestra empresa - unidades de producción. www.minsur.com.pe/unidadesproduccion.htm (Visitado el 05-04-2011).

[9] Phelke, R. (1973). Unit processes of extractive metallurgy. American Elsevier, Nueva York, Estados Unidos.

[10]Pinedo, L. (2009). Planta de fundición y refinación de estaño. Informe de práctica preprofesional. Funsur S.A. Lima.

[11]Rong, A. y Lahdelma, R. (2008). Fuzzy chance constrained linear programming model for optimizing the scrap charge in steel production. European Journal of Operational Research. Vol. 186, N. ${ }^{\circ} 3,953-964$. 